\title{
La utilización de instrumentos de evaluación en Educación Primaria: análisis de caso en centros educativos de la provincia de Valencia (España)
}

The use of evaluation instruments in Primary Education: A case study on schools in the Province of Valencia (Spain)

\author{
Juan Antonio Giménez Beut \\ jantonio.gimenez@ucv.es \\ Francisco José Morales YaGo ${ }^{1}$ \\ fmoralesyago@gmail.com \\ David Parra Camacho \\ david.parra-camacho@uv.es \\ Universitat de València, España
}

\section{Resumen:}

El objetivo de la investigación presentado en este artículo es el conocer cuáles son los instrumentos de evaluación más empleados entre el profesorado de educación primaria en la provincia de Valencia. Este objetivo inicial pretende, en posteriores investigaciones, compararse con las metodologías que estos profesores manifiestan en su docencia. Se presentan los resultados de una encuesta aplicada a dichos profesores, integrando aspectos sobre el proceso evaluativo, identificando los instrumentos de evaluación utilizados y su frecuencia de aplicación dependiendo básicamente del tipo área de aprendizaje desarrollado. En términos de frecuencia, el profesorado reconoce que las pruebas objetivas son las más usadas, prefiriendo instrumentos de evaluación que les permitan demos-

\begin{abstract}
:
This study aims to know the most commonly used evaluation instruments by Primary Education teachers in the Province of Valencia. This aim, in subsequent studies, will be compared with the methodologies these teachers use in their classes. Data were obtained through a survey completed by these teachers regarding aspects of the evaluation process, identifying the evaluation instruments used and their frequency of application depending basically on the type of subject. In terms of frequency, teachers recognize that objective tests are the most frequently used instrument, preferring evaluation instruments that activate more than one cognitive ability, foster personalized teaching and take into account students' diversity when it comes to the teaching of subject content.
\end{abstract}

1 Dirección para correspondencia (correspondence address):

Francisco José Morales Yago. C/Colón n 44, 2º B. 30510 Yecla, Murcia (España) 
La utilización de instrumentos de evaluación en Educación Primaria: análisis de caso en centros educativos de la provincia de Valencia (España)

Juan Antonio Giménez Beut, Francisco José Morales Yago y David Parra Camacho

trar más de una habilidad cognitiva y que a través de los mismos accedan tanto a la temática a tratar teniendo presente la posible diversidad de alumnado como a la búsqueda de una enseñanza personalizada. El profesorado considera que los instrumentos más eficaces para el aprendizaje del alumnado son los de desempeño en donde la prevalencia del examen escrito juega un papel porcentual elevado en el conjunto de la nota final otorgada. Por último, esta investigación deja abierta la invitación a continuar profundizando en la búsqueda de técnicas y métodos que contribuyan a una evaluación del alumnado cada vez más personalizada, justa y equitativa, pudiendo con ello mejorar las prácticas docentes en pos de la mejora de los procesos de enseñanza-aprendizaje. Descubrir las acciones del profesorado frente a los procesos evaluativos podrá desvelar información importante respecto a lo realizado antes, durante y después de las evaluaciones.

\section{Palabras clave:}

Rúbricas; exámenes; porfolio; exposiciones; proyectos; autoevaluación; coevaluación; trabajos.
Teachers consider that the most effective instruments for student learning are those in which the written exam is assigned a high percentage of the final mark. Finally, this study encourages further research to continue searching for techniques and methods that can contribute to student evaluation models which are increasingly fairer and more personalized and equitable. In this vein, teaching practices will improve leading to better teaching-learning processes. Discovering teachers' actions regarding evaluative processes can reveal important information in relation to what was done before, during and after evaluations.

\section{Key words:}

Rubrics; exams; portfolio; exhibitions; Projects; self appraisal; co-evaluation; jobs.

\section{Résumé:}

L'objectif de la recherche présentée dans cet article est de déterminer quels sont les instruments d'évaluation les plus utilisés par les enseignants de l'enseignement primaire dans la province de Valence. Cet objectif initial est destiné, dans des recherches ultérieures, à être comparé aux méthodologies que ces enseignants utilisent dans leur enseignement. Les résultats d'une enquête appliquée à ces enseignants sont présentés, intégrant des aspects sur le processus d'évaluation, identifiant les instruments d'évaluation utilisés et leur fréquence d'application dépendant essentiellement du type de domaine d'apprentissage développé. En termes de fréquence, les enseignants reconnaissent que les tests objectifs sont les plus utilisés, préférant les instruments d'évaluation qui leur permettent de démontrer plus d'une capacité cognitive et qui leur permettent d'accéder à la fois à la matière à traiter, en tenant compte de la diversité possible des élèves et de la recherche d'un enseignement personnalisé. Le corps enseignant considère que les instruments les plus efficaces pour l'apprentissage des étudiants sont ceux de la performance, où la prévalence de l'examen écrit joue un rôle important dans la note finale attribuée. Enfin, cette recherche laisse ouverte l'invitation à poursuivre l'approfondissement dans la recherche de techniques et de méthodes qui contribuent à une évaluation des élèves de plus en plus personnalisée, juste et équitable, pouvant ainsi améliorer les pratiques d'enseignement afin d'améliorer les processus d'enseignement-apprentissage. Découvrir les actions de l'enseignant dans le processus d'évaluation peut révéler des informations importantes sur ce qui a été fait avant, pendant et après les évaluations.

\section{Mots clés:}

Rubriques; examens; portfolio; expositions; projets; auto-évaluation; co-évaluation; missions. 
Fecha de recepción: 26-09-2019

Fecha de aceptación: 17-01-2020

\section{Introducción}

Los instrumentos de evaluación son el medio con el cual el profesorado puede controlar el proceso del aprendizaje al registrar y obtener la información necesaria para verificar los logros o dificultades del alumnado en función de unos objetivos inicialmente establecidos (López e Hinojosa, 2018). Según sus necesidades se podrían agrupar básicamente en dos tipos, los dedicados a la evaluación de la enseñanza y los dedicados a la evaluación de aprendizajes.

La evaluación sumativa se desarrolla en el día a día de la Escuela, y suele ser estar poco valorada por el alumnado, lo que nos Ileva hacia el interés de una mayor participación y sensibilización por parte de ellos para que pasen hacia un papel más activo. En general, el curriculum plantea contenidos que van ampliándose en la medida que el alumnado va pasando de curso en un proceso constructivista del proceso de enseñanza-aprendizaje (Arribas, 2017), que puede vincularse hacia el excesivo apoyo en la memoria, lo que repercute en la extensión de una metodología repetitiva, que acarrea un mayor grado de desmotivación en el proceso de enseñanza-aprendizaje., La evaluación de dichos contenidos (Ruay, Jara, y López, 2013), en este contexto, resulta igualmente compleja y árida, utilizando instrumentos de evaluación basados en pruebas escritas con enunciados formales y alejados de sus vivencias e intereses (Salinas, 2002), lo que detrae una comunicación poco formativa basada en listados o boletines de calificaciones que basan su información más en un resultado numérico que en una información analítica sobre el resultado de la evaluación (Rosales, 2014).

Estas formas de evaluar pueden estar relacionadas con un concepto de enseñanza por parte del profesorado que pueden asociarse a vivencias anteriores de los mismos, aunque ya desde hace décadas se han planteado propuestas interesantes para la transformación de estas ideas sobre enseñanza y evaluación (Coll y Martín, 1993; Alonso et al., 1995), la problemática sigue existiendo en las aulas al organizar la evaluación del alumnado desde una perspectiva equitativa y organizacional (Cardona y Zambrano, 2014). Es imprescindible, cambiar de mentalidad or- 
ganizativa con el alumnado, enseñando y evaluando en función de los procesos y cambios que han sobrevenido a su realidad cotidiana. En esta línea, y para que la integración de la evaluación en el contexto general del proceso de planificación de la enseñanza sea real y asumida con naturalidad por alumnos y profesores, es necesario ampliar su sentido, valorando aparte de la función social, las funciones de tipo pedagógico de la evaluación (Jorba y Sanmartí, 1993; Menoyo, 1995; Sanmartí, 2007), concienciándoles de que se puede aprender mientras se evalúa y que es necesario evaluar a los distintos agentes que intervienen en el proceso de enseñanza: alumnado y profesorado.

Por este motivo, se ha planteado averiguar cuáles son los instrumentos de evaluación más utilizados entre el profesorado de educación primaria de la provincia de Valencia.

\section{Marco teórico}

En todo proceso de evaluación se requiere de una recogida de información respecto del objeto a evaluar. En materia educativa, la recopilación de evidencias se realiza principalmente a través de instrumentos de evaluación, que pueden ser definidos como todo aquello que nos permite obtener información respecto a la adquisición y grado de logro de un aprendizaje de los estudiantes (Castillo, 2003; Pimienta, 2008). Estos instrumentos deben ser de óptima calidad, pues solo así puede asegurarse la obtención de evidencias fiables sobre el aprendizaje de los estudiantes. La información proporcionada a través de la aplicación de los instrumentos de evaluación clarifica el proceso de toma de decisiones y ello permite mejorar y desarrollar con éxito los procesos de enseñanza y aprendizaje (Sanmartí, 2007).

Encontramos diversos tipos y clasificaciones de instrumentos de evaluación. Cabrera (2000) insiste en que lo más usual es distinguir entre cuantitativos (pruebas de aprendizaje tipo test u objetivas, cuestionarios, procedimientos de observación sistematizados y estructurados) y cualitativos (pruebas de preguntas abiertas y de ejecución no estandarizadas, entrevistas abiertas, proceso de observación participantes, análisis de contenidos, procedimientos grupales como grupos de discusión, grupo nominal, foros, etc.). Haladyna (1994) y Scheerens, Glas y Thomas (2003), distinguen entre instrumentos de selección de respuesta (donde 
la respuesta correcta es seleccionada dentro de la pregunta), instrumentos de construcción de respuesta (donde la respuesta debe ser construida por los estudiantes) e instrumentos de desempeño (en donde las tareas de evaluación son similares a situaciones de la vida real). Por su parte, Miller, Linn y Gronlund (2013) dividen entre instrumentos objetivos y de desempeño. Otros autores (Jiménez, 2019), diferencian entre criterios de evaluación, pruebas de evaluación, criterios de calificación e instrumentos de calificación.

Se evidencia que la evaluación también queda condicionada por la metodología de enseñanza implementada en el aula. Por todo esto en la actualidad se plantea con creciente interés el análisis de los procesos de evaluación a través de diversas propuestas al respecto (Alonso Sánchez, 1996; Rodríguez Barreiro, 1996, Przesmycki, 2000, Bonilla y López, 2005 y Sanmartí, 2007), como paso importante para avanzar en la aplicación de innovaciones y mejoras en el proceso de enseñanza (Gargallo, 2017), recogiendo el primigenio planteamiento de Barr y Tagg en 1995, y las aportaciones de un gran número de investigadores, planteó dos grandes paradigmas educativos que determinan a su vez la evaluación. Se trata de un primer modelo en la enseñanza que realiza al profesor como principal fuente de conocimiento cuya misión es enseñar a los alumnos y que tiene como forma de evaluar la enseñanza tradicional. El otro modelo estaría centrado en el aprendizaje y en el modo como los alumnos construyen los significados. Este segundo modelo requiere de una evaluación formativa.

En este modelo la enseñanza y la evaluación se centra en promover el aprendizaje del alumno, su autonomía y el desarrolla de habilidades metacognitivas de autorrealización y conducción del propio proceso de aprendizaje (...) el uso de una evaluación significativa planteada como oportunidad de aprendizaje, con utilización de diferentes fuentes de recogida de información, que devuelve feedback a los alumnos (Hernández, 2012, p.22)

Siguiendo el alineamiento constructivo de Bigss (2015), es necesario hacer coherentes entre sí todos los elementos del currículo. En este caso es motivo de reflexión comprobar si la actual novedad por introducir metodologías diferentes es fruto de una verdadera revisión o son tan solo una renovación superficial. La evaluación debe ser coherente con las metodologías empleadas en los procesos instructivos del aula, y es nece- 
sario evaluar la eficacia de estas metodologías con evidencias claras de su efecto en los resultados en el aprendizaje de los alumnos.

La innovación educativa actual se asienta sobre investigaciones de eficacia validada en muy diferentes contextos educativos. Pero una de las constantes en su implementación, es la necesidad de vincular la introducción de nuevas metodologías de aula a la evaluación de la calidad de aprendizaje que producen (Pellicery Ortega, 2009, p.6)

Los instrumentos que utilizamos para evaluar nos permiten determinar los aprendizajes conseguidos, lo que a su vez revierte -o debería hacerlo- en la propia mejora de la práctica docente. Pero, si elegimos instrumentos de evaluación incoherentes con las metodologías empleadas obtendremos resultados equivocados.

Tratando de contribuir a este proceso de reformulación de la evaluación, es importante conseguir un mayor proceso de diálogo y comprensión en la evaluación (Santos, 1993). Se debe perseguir el logro de una fluidez en la información en la doble dirección profesor-alumnos y en todas las etapas del proceso como serían los criterios claros de evaluación, especificación de las técnicas e instrumentos aplicados y realización de una valoración integradora de los resultados.

En la evaluación formativa, es necesaria la adaptación del proceso didáctico a los progresos y problemas de aprendizaje del alumnado a través de la regulación pedagógica (Sanmarti y Sorba, 1995; Perrenoud, 2001), de tal forma que podamos corroborar que la evaluación es útil para aprender y tiene la finalidad reguladora en el proceso de aprendizaje. Se pretende conseguir una óptima gestión de las dificultades halladas (Astolfi, 1999), cambiando así la concepción sobre el papel en la no consecución de los objetivos previstos la consolidación de los éxitos. Se trata, en definitiva, de potenciar la función pedagógica de la evaluación a través de aspectos formativos tanto para los alumnos (reforzar su proceso de aprendizaje) como para el profesor (mejorar su proceso de enseñanza) y competencial (Ruay y Garcés, 2015). Se busca por tanto una evaluación motivadora (Alonso, 2005), en donde habrá que resaltar los logros, hacerse corresponsable de sus fracasos, haciendo ver que se puede aprender en el proceso de evaluación si son utilizados instrumentos y técnicas de evaluación que, sin perder su eficacia, sean más atractivos y relacionados con su entorno cotidiano y así poder adaptar el modelo 
de evaluación propuesto al contexto real en donde se quiere poner en práctica alcanzado las competencias pretendidas.

La capacidad de una autoevaluación por parte de los alumnos se ha puesto en duda, porque parece más fiable que lo realice el propio profesor, tal vez por pensar que el alumnado está sometido a «presiones» relacionadas con su calificación de la asignatura, que les hace ser parciales y subjetivos al valorar la labor docente de sus profesores. La evaluación del profesorado aparece como un buen recurso para valorar su proceso de enseñanza y su papel como docente, aunque no manifiestan el posible inconveniente de subjetividad, el cual se reduce cuando interviene un observador ajeno al proceso de enseñanza. Por otro lado, es interesante que algunos autores citen la necesidad de evaluar los proyectos educativos, los materiales y recursos utilizados para la enseñanza. Se observa que aparece poco el tema de la coevaluación, dejando sobre todo su función evaluadora para diagnosticar la labor de su profesor y el proceso de enseñanza seguido. Tal vez se deba a que están más acostumbrados a rellenar cuestionarios de evaluación de sus profesores, mientras que sus experiencias de coevaluación son más bien anecdóticas.

El examen escrito sigue siendo la prueba clásica más destacada para valorar a los alumnos, de tal forma que prácticamente en todas las propuestas de evaluación que citan, incluso las más novedosas, siempre aparece de forma prioritaria la necesidad de realizar también algún examen escrito, aspecto que resulta curioso pues es precisamente este tipo de pruebas escritas las que más suelen estar relacionadas con la evaluación que ellos perciben como «desagradable», no motivadora, poco comunicativa (listado de calificaciones y «miedo» a consultas de exámenes) y, por supuesto, poco o nada formativa. Resulta casi anecdótico que el examen oral (como alternativa al escrito) sea citado por pocos alumnos. Por otro lado, destaca el que apuesta por valorar significativamente el trabajo de clase y el seguimiento continuo del aprendizaje de los alumnos, aunque no concretan de qué forma hacerlo (el profesorado habla de observación o diario de clases pero no de qué metodología a seguir para realizar dicha observación). A la vista de estos resultados es conveniente proporcionales el conocimiento y aplicación de una diversidad de instrumentos de evaluación para ser utilizados en función del tipo y naturaleza de los contenidos a evaluar.

El porcentaje más alto de la muestra relaciona la evaluación con la función exclusiva de comprobación de adquisición de contenidos, re- 
lación que también era mayoritaria en su experiencia como alumnos. No obstante, destacamos que se apuesta por una evaluación relacionada con aspectos formativos (función pedagógica de la evaluación). Los aspectos formativos aparecen también para el caso del profesor, de tal forma que se habla de utilizar los resultados de evaluación para mejorar el proceso de enseñanza. Faltaría comprobar, en el futuro ejercicio de su docencia, hasta qué punto dan sentido real a estos aspectos formativos citados para el proceso de evaluación. Aportaciones interesantes de evaluación formativa, y también comunicativa y motivadora, son los informes de evaluación aportados por Rodríguez (1996) para informar a los alumnos los resultados de dicho proceso, o el uso del portafolios del alumno como instrumento de evaluación (Klenowski, 2005). El objetivo del trabajo planteará el porcentaje que otorga el profesorado de educación primaria a cada uno de los diversos instrumentos de evaluación utilizados.

\section{Método}

\subsection{Participantes}

La obtención de la muestra se realizó previo contacto telefónico con los directores de los centros y la obtención del permiso correspondiente para visitarlos con el posterior envío de cuestionarios a centros educativos localizados en la provincia de Valencia. De todo el listado facilitado por la Consejería de Educación (http://www.ceice.gva.es/es/web/centrosdocentes), finalmente participaron 54 centros escolares de la provincia de Valencia valorando el porcentaje de importancia en la calificación final de diferentes áreas de la educación Primaria que otorgaban a diversos instrumentos de evaluación. Se consultó un 3,76\% (N=17) del total de centros públicos ordinarios de la provincia de Valencia (452) y un $16,97 \%(\mathrm{~N}=37)$ del total de centros privados concertados de la provincia de Valencia (218) en todos los cursos de la etapa de educación primaria, es decir, desde $1^{\circ}$ hasta $6^{\circ}$ de primaria. También se consultó las siguientes áreas del curriculum: lengua castellana, matemáticas, ciencias naturales, ciencias sociales y lengua extranjera. 


\subsection{Instrumento}

El instrumento estaba formado por diversas cuestiones en las que se pedía que cada centro indicase el porcentaje que se otorgaba a cada instrumento de evaluación en la calificación final de cada área según el curso académico. Los instrumentos que se indicaron fueron los siguientes: prueba escrita corta, prueba escrita de desarrollo, examen oral, trabajos individuales, trabajos grupales, proyecto de investigación, exposiciones orales, portafolios, escala de valoración o rúbrica, autoevaluación, coevaluación, actividades (libreta) y otros instrumentos.

\subsection{Procedimiento}

El muestreo utilizado fue de tipo intencional no probabilístico o de conveniencia. Para proceder a la recogida de datos se entregó una ficha directamente a los profesores en las que debían cumplimentar los porcentajes que emplean para las cinco áreas nombradas. Se les presentaron diferentes herramientas de evaluación extraídas de los autores de dejando abierta la posibilidad de añadir otras particulares. Estas fichas fueron entregadas y recogidas entre los meses de mayo de 2018 y febrero de 2019.

\subsection{Análisis estadísticos}

Para realizar los análisis estadísticos se utilizó el programa estadístico para ciencias sociales SPSS v. 23. En primer lugar, se analizaron los estadísticos descriptivos a partir de las puntuaciones medias de los porcentajes de cada instrumento de evaluación por asignatura y ciclo de educación primaria para el conjunto de colegios analizados.

Para comprobar si existían diferencias a nivel estadísticamente significativo $(p<0,05)$ en las puntuaciones medias de los porcentajes se utilizaron pruebas no paramétricas debido a la ausencia de distribución normal de los datos comprobada mediante la prueba de KolmogorovSmirnov $(p<0,05)$. Por esta razón, la prueba utilizada para comprobar si existen diferencias estadísticamente significativas según el tramo de educación primaria y el tipo de centro educativo (concertado o público) es la U de Mann Whitney. 


\section{Resultados}

\subsection{Análisis descriptivo del porcentaje medio de utilización de los instrumentos de evaluación}

A continuación, se presentan los resultados del estudio, mostrando las puntuaciones medias otorgadas, por los centros educativos entrevistados, a cada instrumento de evaluación tanto a nivel general de las seis áreas consultadas (lengua castellana y literatura, matemáticas, inglés, ciencias de la naturaleza y ciencias sociales) como según el tipo de asignatura.

En la figura 1 se observan las puntuaciones medias de cada instrumento de evaluación para todas las áreas y cursos consultados (desde $1^{\circ}$ de educación primaria hasta $6^{\circ}$ de educación primaria). Como se puede observar la prueba escrita es el instrumento que representa un porcentaje más destacado $(38,6 \%)$ a la hora de calificar a los alumnos, seguido de los trabajos individuales $(14,4 \%)$ y de los trabajos grupales $(10,6 \%)$. En cambio, es importante resaltar la escasa presencia de instrumentos como la autoevaluación $(1,7 \%)$ o la coevaluación $(1,3 \%)$ en los procesos de evaluación en educación primaria en la muestra de centros consultada.

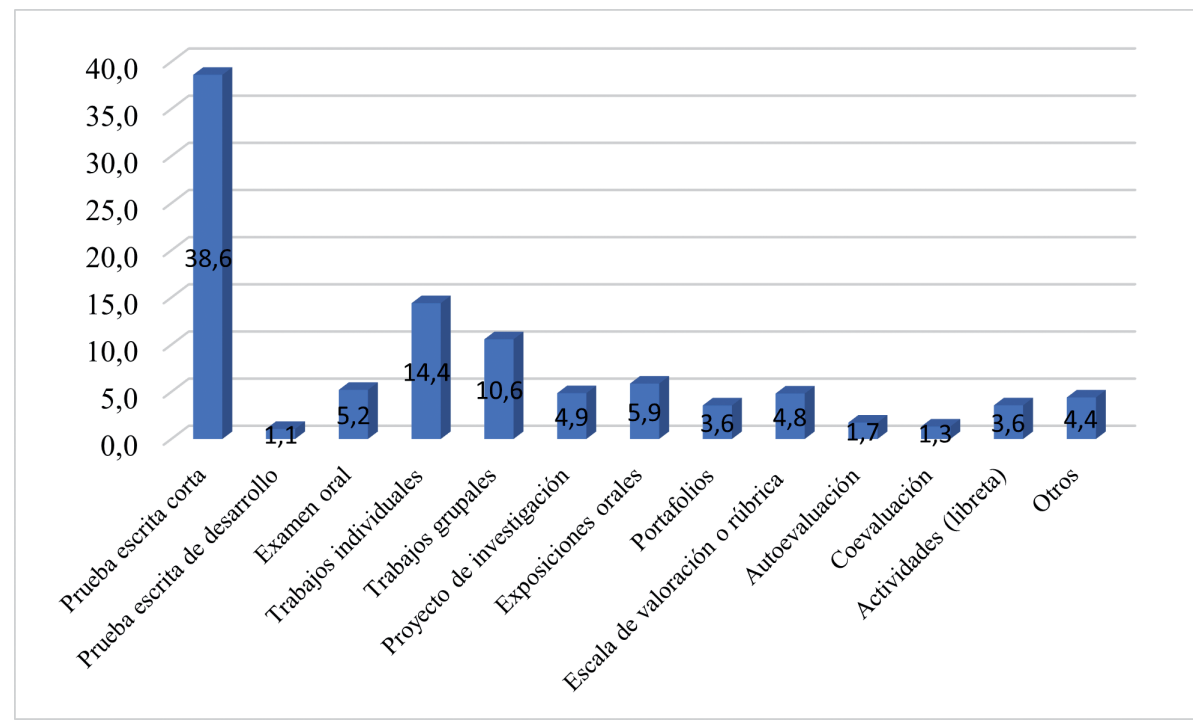

Figura 1. Porcentaje medio de los instrumentos de evaluación para la etapa de educación de primaria en el conjunto de centros analizado. 
En la figura 2 se observan las puntuaciones medias de cada instrumento de evaluación según el área para todos los cursos consultados (desde $1^{\circ}$ de educación primaria hasta $6^{\circ}$ de educación primaria). Tal y como se puede apreciar el instrumento de evaluación que representa un porcentaje más elevado en todas las áreas es el del examen escrito breve, siendo el área de matemáticas la que más destaca esta herramienta de evaluación (45,5\%). A más distancia se encuentran los trabajos individuales como instrumento de evaluación empleado en las áreas de lengua castellana y literatura (15,3\%), matemáticas $(13,8 \%)$ y ciencias naturales (12,9\%). En el caso de ciencias naturales y ciencias sociales se observa que los trabajos grupales e individuales muestran porcentajes bastante similares en cuanto a relevancia dentro de la calificación final de la asignatura, destacando ligeramente por encima los trabajos grupales en el caso del área de ciencias sociales $(13,1 \%)$. Para el área de inglés se observa que el examen oral es la segunda herramienta de evaluación más importante para los docentes en esta área $(12,6 \%)$.

La autoevaluación y la coevaluación tienen una escasa presencia, siendo inferior al 5\% del total de la calificación en todas las áreas consultadas. Por último, destacar que los proyectos de investigación ocupan el cuarto lugar como instrumento de evaluación en cuanto a importancia en la calificación final se refiere en las áreas de ciencias naturales $(7,9 \%)$ y ciencias sociales $(7 \%)$. 
La utilización de instrumentos de evaluación en Educación Primaria: análisis de caso en centros educativos de la provincia de Valencia (España)

Juan Antonio Giménez Beut, Francisco José Morales Yago y David Parra Camacho

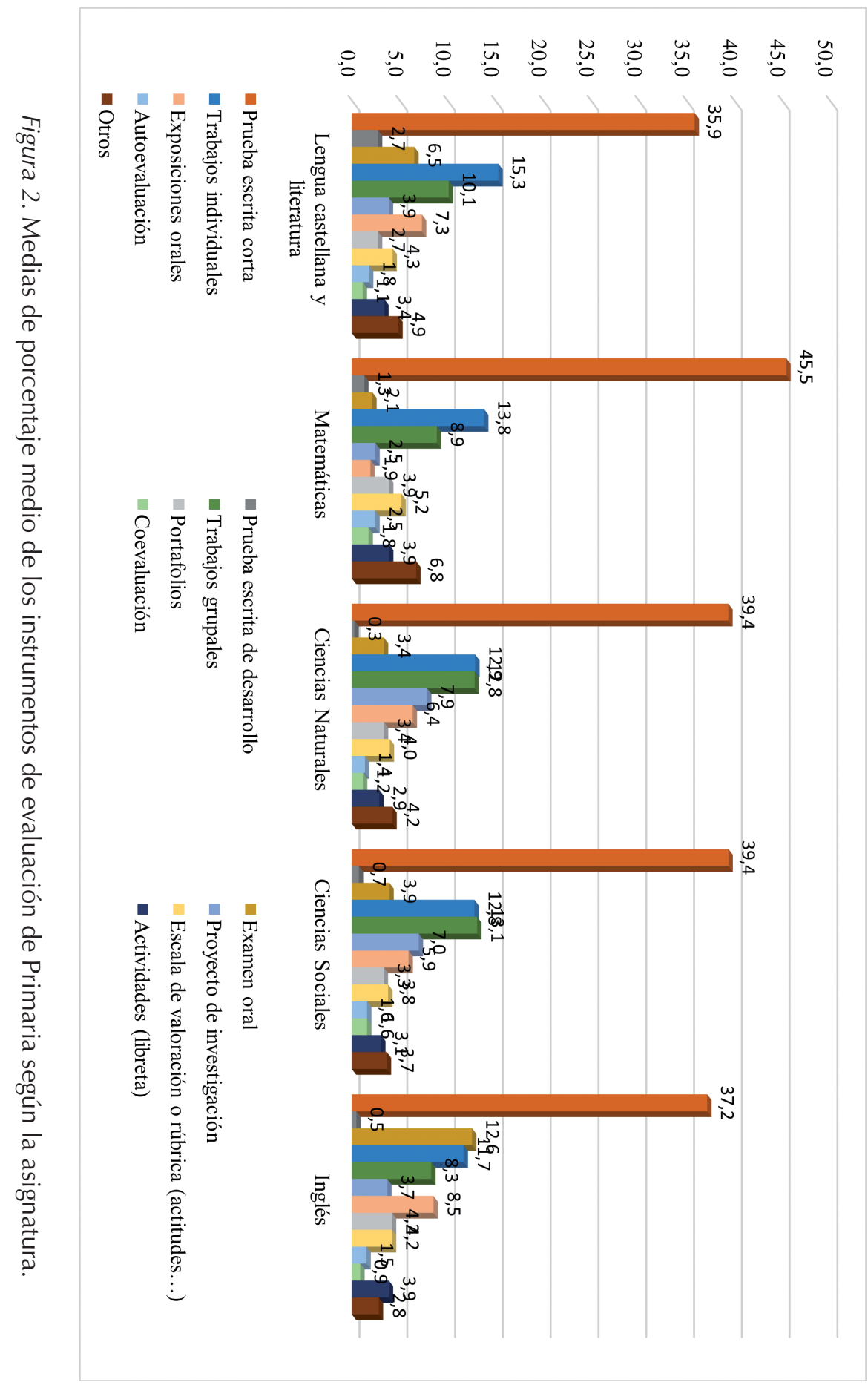




\subsection{Análisis de la influencia del tramo educativo sobre el porcentaje medio de los instrumentos de evaluación según el área}

En la tabla 1 se muestran los resultados del porcentaje medio otorgado por los centros consultados a cada instrumento de evaluación según la asignatura y el tramo de la etapa educación primaria. En el primer tramo se incluyen los cursos de $1^{\circ}$ a $3^{\circ}$ de educación primaria y en el segundo tramo se incluyen los cursos de $4^{\circ}$ a $6^{\circ}$ de primaria.

Tal y como se puede apreciar, no existen diferencias estadísticamente significativas entre la mayoría de los porcentajes medios para cada instrumento en función del tramo. Tan solo se aprecian diferencias estadísticamente significativas $(p<0,05)$ entre los porcentajes medios del primer tramo $(M=13,57)$ y el segundo tramo $(M=9,26)$ para los trabajos individuales en el área de inglés; y para la categoría de otros instrumentos de evaluación en el caso de las áreas de ciencias sociales y ciencias naturales.

De forma general, se puede observar que el porcentaje medio otorgado a las pruebas de exámenes escritos de desarrollo incrementa en todas las áreas su importancia en el segundo tramo, aunque las diferencias no son estadísticamente significativas. La tendencia inversa se observa en el caso de otros instrumentos de evaluación cuya importancia en la calificación final disminuye en el segundo tramo, como, por ejemplo, los trabajos individuales. 


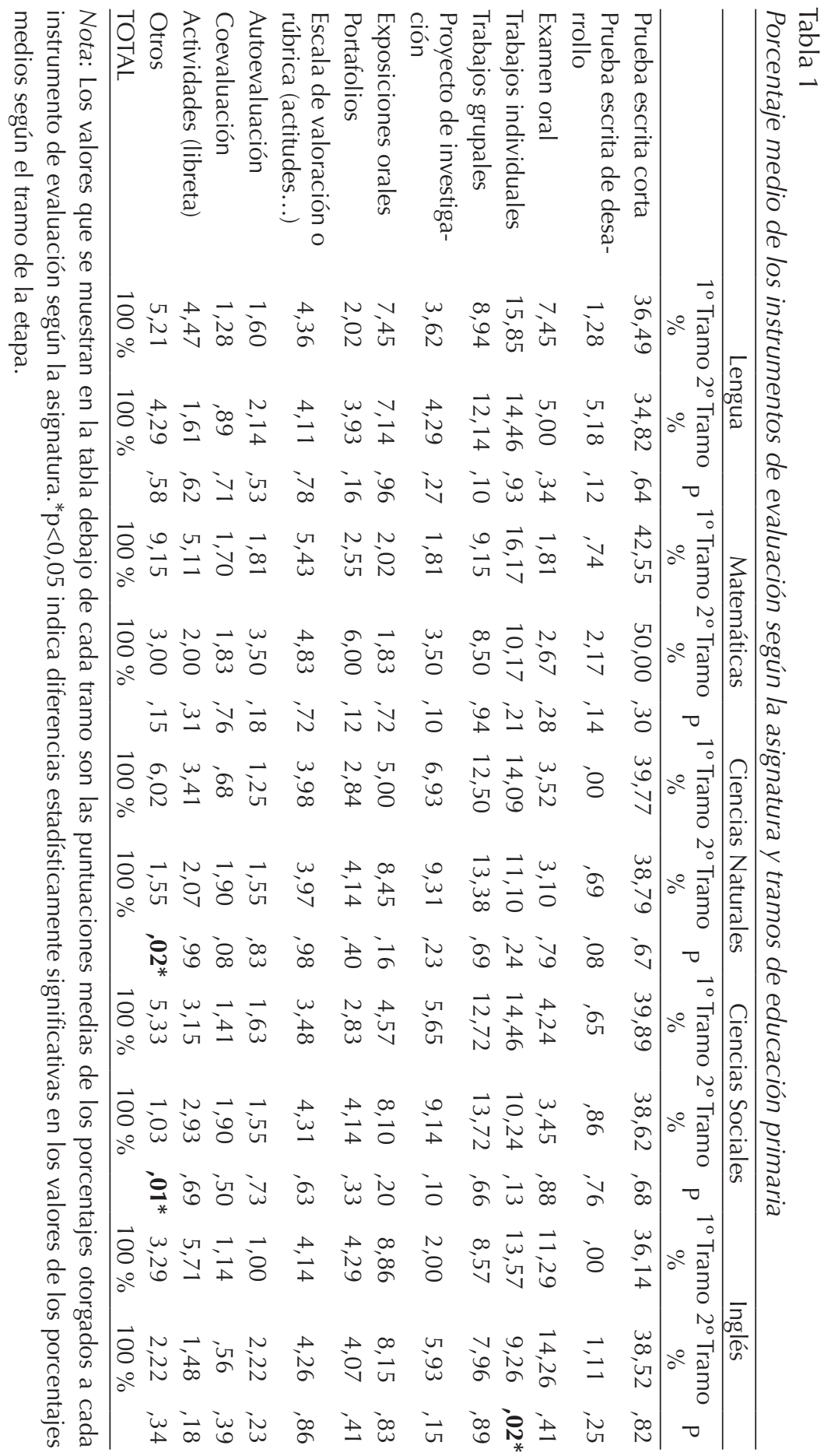




\subsection{Análisis de la influencia del tipo de centro educativo sobre el porcentaje medio de los instrumentos de evaluación según el área}

En la tabla 2 se muestran los resultados del porcentaje medio otorgado por los centros consultados a cada instrumento de evaluación según la asignatura y el tipo de centro (concertado o público). Como se puede apreciar, existen diferencias estadísticamente significativas $(p<0,05)$ entre los porcentajes medios de la prueba escrita para las áreas de matemáticas, ciencias sociales y ciencias naturales en función del tipo de centro. En todos los casos se observa que el porcentaje medio de la prueba escrita es inferior en el centro público que en los centros privados. Por ejemplo, en el caso del área de matemáticas el porcentaje medio para esta prueba de evaluación en los centros públicos es de 35,23\%, mientras que en los centros concertados es del 49,55\%; para el área de ciencias naturales es de $29,55 \%$ y $43,63 \%$, respectivamente; mientras que para el área de ciencias sociales es de $30,91 \%$ y $42,92 \%$, respectivamente. Incluso en las áreas en las que no se han observado diferencias estadísticamente significativas entre los porcentajes medios, como es el área de inglés y la de lengua castellana y literatura, se constata la misma tendencia.

También se observan diferencias estadísticamente significativas $(p<0,05)$ entre los porcentajes medios de los trabajos grupales según el centro. En el caso de los centros públicos tiene mayor importancia que en los centros concertados. Así se puede apreciar en los porcentajes medios del área de lengua castellana y literatura $(M=8,80$ para los centros concertados y $M=13,57$ para los centros públicos); el área de matemáticas $(M=7,91$ para los centros concertados y $M=11,36$ para los centros públicos); o el área de ciencias naturales ( $M=11,73$ para los centros concertados y $M=15,45$ para los centros públicos).

Por otro lado, en el caso de los proyectos de investigación se observaron diferencias estadísticamente significativas en los porcentajes según el tipo de centro para el área de ciencias naturales $(M=6,27$ para los centros concertados y $M=11,59$ para los centros públicos), siendo los centros públicos los que otorgan mayor importancia a este instrumento.

Por último, se aprecian diferencias estadísticamente significativas $(p<0,05)$ en la coevaluación según el tipo de centro en el área de matemáticas, aunque el porcentaje medio es bastante reducido en los dos tipos de centros consultados $(M=1,27$ en los centros concertados y $M=2,95$ en los centros públicos). 
La utilización de instrumentos de evaluación en Educación Primaria: análisis de caso

en centros educativos de la provincia de Valencia (España)

Juan Antonio Giménez Beut, Francisco José Morales Yago y David Parra Camacho

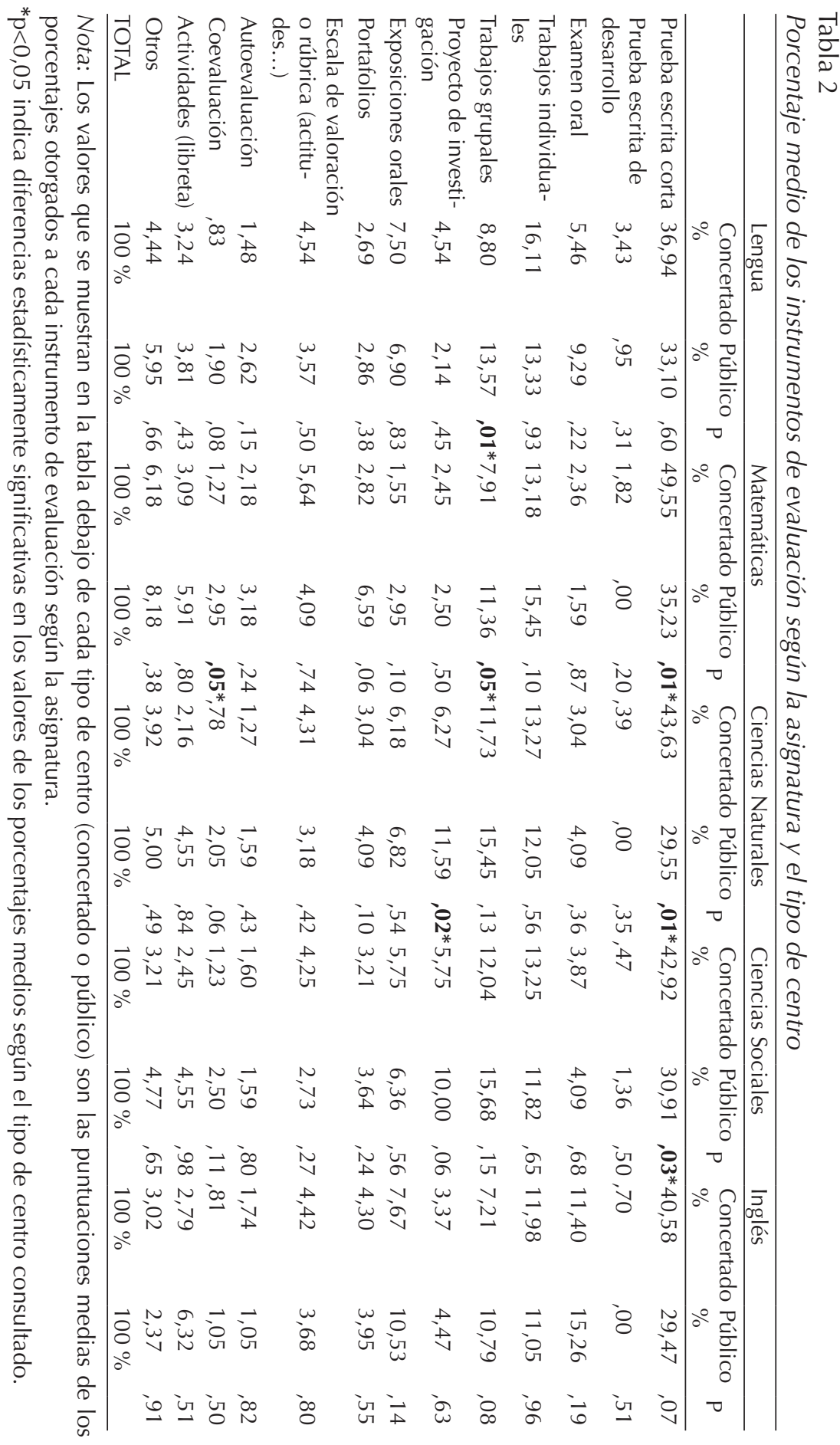




\section{Conclusiones y Prospectiva}

Aunque existen variados instrumentos de evaluación y el profesorado ha ido incorporándolos en su tarea docente, se ha comprobado que el instrumento prevalente actualmente continua siendo la tradicional prueba escrita con un $38,6 \%$, llegando al $45,5 \%$ en el caso del área de matemáticas.

Si bien es cierto que habría que contrastar la forma de preguntar en esta misma prueba escrita, en principio este instrumento se clasificaría dentro del paradigma centrado en la enseñanza (Gargallo, 2017).Como se exponía en el marco teórico, se trata de una evaluación "no motivadora, poco comunicativa y poco formativa". No se busca rechazar este instrumento de evaluación, sino de reducir el actual peso que tiene en la evaluación global de los alumnos. También se ha evidenciado la escasa presencia de la autoevaluación $(1,7 \%)$ y la coevaluación (1,3\%). Esto coincide con las investigaciones presentadas en el marco teórico que planteaban que eran consideradas como poco fiables al ligarlo a la calificación final y la subjetividad que esto conlleva.

Los trabajos grupales son empleados de manera más equivalente en las ciencias sociales y naturales. Sin embargo, los exámenes orales son poco utilizados por las diferentes áreas. Tan sólo es relevante para el inglés, que la utiliza en segundo lugar $(12,6 \%)$. Esto es coherente porque esta área se orienta en torno a las cuatro habilidades básicas y precisas de su expresión y comprensión oral. Los proyectos de investigación son también propios del paradigma del aprendizaje. Son poco relevantes en las áreas, aunque en el caso de ciencias naturales $(7,9 \%)$ y ciencias sociales $(7 \%)$ ocupan un cuarto lugar.

Las escalas de valoración $(4,8 \%)$ y el seguimiento de las tareas diarias a través de la libreta $(3,6 \%)$ tienen un valor relativamente pequeño, lo que contrasta con el interés por realizar un seguimiento de las tareas diarias de los alumnos.

Como se ha expuesto anteriormente, el portafolios es un instrumento formativo, comunicativo y motivador (Klenowski, 2005). Sin embargo, sólo ha obtenido una puntuación de un 3,6\%.

Por otro lado, no se han encontrado diferencias estadísticamente significativas entre la mayoría de los porcentajes medios para cada instrumento en función del tramo educativo (de $1^{\circ}$ a $3^{\circ}$ y de $4^{\circ}$ a $6^{\circ}$ ). Tan solo entre los porcentajes medios del primer tramo $(M=13,57)$ y 
el segundo tramo $(M=9,26)$ para los trabajos individuales en el área de inglés.

En cuanto al tipo de centro (público o privado/concertado) existen diferencias significativas $(p<0,05)$ entre los porcentajes medios de la prueba escrita para las áreas de matemáticas, ciencias sociales y ciencias naturales.

También, encontramos que el porcentaje medio de la prueba escrita es inferior en el centro público que en los centros privados. En cuanto a las matemáticas el porcentaje medio para esta prueba en los centros públicos es de $35,23 \%$, mientras que en los centros concertados es del 49,55\%; para el área de ciencias naturales es de 29,55 y 43,63, respectivamente; mientras que para el área de ciencias sociales es de 30,91 y 42,92, respectivamente.

Podemos afirmar pues que, a tenor de los resultados extraídos, se encontraría una mayor tendencia a instrumentos de evaluación vinculados al paradigma del aprendizaje en los centros públicos que en los privados.

Hemos conocido las tendencias de los profesores de la provincia de Valencia respecto a los instrumentos de evaluación más utilizados. Atendiendo a la clasificación en enfoque de enseñanza y aprendizaje (GargaIlo, 2017) podríamos observar que sigue existiendo una predominancia hacia el primer enfoque con el destacado instrumento del examen escrito. Con una mayor presencia en los centros privados que en los públicos. Sería ahora procedente comprobar qué metodologías de enseñanza-aprendizaje han utilizado estos profesores. De esta forma podríamos determinar si existen discrepancias entre la metodología de enseñanza y los instrumentos de evaluación. Si esto fuera así, podríamos afirmar que muchos centros educativos han experimentado un cambio superficial y no profundo, dando lugar a una cierta distorsión curricular (Biggs, 2015). Como prospectiva sería importante profundizar en la forma en que se utilizan estos instrumentos y la combinación de estos para alcanzar las competencias esperadas.

Finalmente constatamos en este trabajo un cierto desfase entre la evaluación que ha existido en la aplicación de nuevas metodológicas en el aula como serían la utilización de técnicas cooperativas, aprendizaje basados en proyectos y en problemas, utilización de las Tics o aprendizaje servicio y los instrumentos de evaluación que es necesario aplicar para equiparar metodologías activas con una evaluación más globalizadora que pueda dar respuesta a los métodos de enseñanza-aprendizaje 
La utilización de instrumentos de evaluación en Educación Primaria: análisis de caso en centros educativos de la provincia de Valencia (España) Juan Antonio Giménez Beut, Francisco José Morales Yago y David Parra Camacho

planteados y hacer frente a la necesaria atención a la diversidad que va aumentando en nuestras aulas del siglo XXI.

\section{Referencias}

Alonso, J. (2005).Motivar en la Escuela, motivar en la familia: claves para el aprendizaje. Madrid: Morata.

Alonso, M., Gil, D. y Martínez, J. (1995).Concepciones docentes sobre la evaluación en la enseñanza de las Ciencias. Alambique, 4, 6-15.

Alonso Sánchez, M. (1996).Evaluar no es calificar. La evaluación y la calificación en una enseñanza constructivista. Investigación en la Escuela, 30, 15-25.

Astolfi, J.P. (1999). El error, un medio para enseñar. Sevilla. Investigación y enseñanza, 15. Arribas, J.M. (2017), La evaluación de los aprendizajes, problemas y soluciones. Rev. De Curriculum y formación del profesorado, 21, $\mathrm{n}^{\circ} 4$.

Biggs, J. (2015). Calidad del aprendizaje universitario. Madrid: Narcea

Bonilla, M. X. y López, A. (2005). ¿Las concepciones de evaluación de los docentes están articuladas con las epistemológicas y de aprendizaje? VII Congreso Internacional Investigación en Enseñanza de las Ciencias. Granada.

Cabrera, F. (2000).Evaluación de la Formación. Serie Didáctica y Organización Escolar, 10. Madrid: Síntesis

Cardona, y Zambrano, R. (2014). Revisión de instrumentos de evaluación del clima organizacional. Rev. Estudios Gerenciales, 30, 184-189

Castillo, S. (2003).Vocabulario de evaluación educativa. Ciudad de México: Pearson.

Coll, C. y Martín, E. (1993).La evaluación del aprendizaje en el currículum escolar. Una perspectiva constructivista. En Coll y otros. El constructivismo en el aula. Barcelona. Grao.

De Pro, A., Hernández, L. y Saura, O. (1997).La compleja tarea de enseñar la ciencia escolar: dificultades para incorporar la actualidad científica al aula. Publicaciones, 25-27, 161-184.

Gargallo, B. (coord.) (2017). Enseñanza centrada en el aprendizaje y diseño por competencias en la universidad. Valencia: Tirant Humanidades.

Haladyna, T. (1994).A research agenda for licensing and certification testing validation studies.Evaluation and the Health Professions, 17(2), 242-56.doi: 10.1177/0163278794017700208

Jiménez, M.A. (Coord.) (2019). Programar al revés. El diseño curricular desde los aprendizajes. Madrid: Narcea

Jorba, J. y Sanmartí, N. (1993). La función pedagógica de la evaluación. Aula de Innovación Educativa, 20, 20-30.

Klenowski, V. (2005).Desarrollo de portafolios para el aprendizaje y la evaluación. Madrid. Nancea.

López, B e Hinojosa, E. (2018).Evolución del aprendizaje y nuevos desarrollos. Ed. Trillas. México. 
La utilización de instrumentos de evaluación en Educación Primaria: análisis de caso en centros educativos de la provincia de Valencia (España)

Juan Antonio Giménez Beut, Francisco José Morales Yago y David Parra Camacho

Menoyo, MP. (1995). La evaluación formativa como instrumento de atención a la diversidad: una experiencia en Secundaria. Alambique, 4, 42-54.

Miller, D., Linn, R. y Gronlund, N. (2013). Measurement and assessment in teaching. Nueva Jersey: Pearson.

Pellicer, C. y Ortega, M. (2009). La evaluación de las competencias básicas. Propuestas para evaluar el aprendizaje. Boadilla del Monte (Madrid): PPC.

Perrenoud, P.H. (2001). Évaluation formative et evaluation certificative: postures con radictoire sou complementaires? Formation professiona llesuisse, 4, 25-28.

Pimienta, J. (2008). Evaluación de los aprendizajes, un enfoque basado en competencias. Ciudad de México: Pearson.

Przesmycki, H. (2000). La pedagogía del contrato. El contrato didáctico en la educación. Barcelona: Grao.

Rodríguez, L.M. (1996). Los informes de evaluación en ciencias. Investigación en la Escuela, 30, 99-110.

Rosales, M. (2015). Proceso evaluativo: evaluación sumativa, evaluación formativa y Assesment su impacto en la educación actual. Libro de Actas del Congreso Iberoamericano de Ciencia, Tecnología, Innovación y Educación. Buenos Aires.

Ruay, R; Jara, P y López, M. (2013). De una evaluación de contenidos a una evaluación de competencias. Redipe. Red Iberoamericana de Pedagogía: Cali, Colombia.

Ruay, R. y Garcés, J.L. (2015). Diseño y construcción de instrumentos de evaluación de aprendizajes y competencias. Readlpe: Colombia

Salinas, D. (2002). ¡Mañana examen! La evaluación: entre la teoría y la realidad. Barcelona: Graó.

Scheerens, J., Glas, C., y Thomas, S. (2003). Educational evaluation, assessment, and monitoring. Lisse: Swets \& Zeitlinger Publishers.

Sanmartí, N. y Jorba, J. (1995). Autorregulación de los procesos de aprendizaje y construcción del conocimiento. Alambique, 4, 59-78.

Sanmartí, N. (2007). 10 ideas clave. Evaluar para aprender. Barcelona: Graó.

Santos Guerra, M.A. (1993). La evaluación: un proceso de diálogo, comprensión y mejora. Málaga: Aljibe 